\title{
Some Kinds of Teas Suppress a Sideration of Diabetes in Streptozotocin-Administered Rats
}

\author{
Masahiro WADA, ${ }^{*}$ Toshichika TAKITA, and Satoshi INNAMI \\ Department of Nutrition, Tokyo University of Agriculture, \\ Setagaya-ku, Tokyo 156, Japan
}

(Received September 7, 1995)

\begin{abstract}
Summary We studied the effects of Maccha and alcohol extracts of Maccha and Mate tea in streptozotocin (STZ)-induced diabetic rats. By the continuous feeding of these substances prior to STZ administration, the elevations of urinary glucose excretion and fasting blood sugar were suppressed, suggesting alleviation of diabetic manifestations. At the same time, the increase in serum lipids, which is a sign of complications in diabetes, was also suppressed; and the concentrations of serum and liver thiobarbituric acid-reactive substance (TBARS) were significantly decreased. The decrease in liver NAD was also suppressed, indicating alleviation of disorders caused by oxidation in the body. The serum uric acid level, which is assumed to elevate in diabetes to compensate for the disorders attributable to oxidation, was significantly lowered. These results suggest that antioxidant components such as the catechins contained in the tested tea leaves act to scavenge STZ-induced radicals and thereby alleviate the disorders caused by oxidation in the body attributable to the formation of radicals.
\end{abstract}

Key Words: diabetes, streptozotocin, Maccha, Mate tea, rat

It has been pointed out that active oxygen and free radicals are closely related to sideration and exacerbation of diabetes and its complications. In the domain of diabetes research, the mechanism is recognized to be as follows: glycosylation of protein by glycation forms free radicals which in turn destroy pancreatic $\beta$ cells $[1$, $2]$. In both insulin-dependent diabetes mellitus (IDDM) and non-insulin-dependent diabetes mellitus (NIDDM), radicals are assumed to be deeply involved in development of the disease. How to suppress glycation and formation of free radicals is therefore a matter of utmost concern.

* To whom correspondence should be addressed. 
Restricting dietary energy intake is generally recommended for diabetic patients. Anderson and Ward [3] reported that a high-carbohydrate, low-fat, and high-fiber diet was effective. As water-soluble dietary fibers suppress elevation of postprandial blood sugar [4] and have an insulin secretion-sparing action $[5,6]$, they are effectively utilized in the diet for prevention and therapy of diabetes.

However, very few studies have been conducted to scientifically elucidate the direct effects of food components on development and morbidity of diabetes. There are only a couple of reports available regarding the decrease in glycosylated protein in the blood [7] and improvement of hyperlipidemia, a complication of diabetes [8], by administration of the antioxidant vitamin E.

The present experiment was designed to clarify if intake of certain kinds of food or food components would suppress manifestations of experimentally induced diabetes with the goals of preventing adult-type diabetes mellitus and improving the clinical conditions of patients with the disease. Their potential supplementary effect on established therapy was also considered.

We took note of tea leaves considered to have antioxidant action in the body, and chose two different species of tea, powdered green tea (Maccha) and Mate tea. We examined the effects of Maccha and alcohol extracts of Maccha and Mate tea leaves on sideration of experimental diabetes.

\section{MATERIALS AND METHODS}

Foods and food components. Maccha (Makinoen Co., Ltd.) manufactured from the leaves of Camellia sinensis (L.) O. Küntze (produce of Shizuoka Prefecture), an alcohol extract of Maccha prepared by adding to Maccha a 4-fold volume of ethyl alcohol and allowing extraction to proceed at room temperature for 7 days, and an alcohol extract of Mate tea leaves produced from Ilex paraguaynensis (produce of the Federative Republic of Brazil) were used as samples.

Animals and composition of diets. Six-week old, male Wistar strain rats (Tokyo Laboratory Animals Co., Ltd., Tokyo) were used for the test. They had been fed the experimental diets for 14 days before they were intraperitoneally administered streptozotocin (STZ) (Wako Pure Chemical Industries, Ltd., Osaka, Japan), which had been previously dissolved in $0.1 \mathrm{M}$ citrate buffered solution ( $\mathrm{pH}$ 4.5 ), at $70 \mathrm{mg} / \mathrm{kg}$ body weight $/ \mathrm{ml}$. STZ is known to cause formation of radicals and induce diabetes in the living body. Following administration of STZ, the rats were housed in metabolic cages and fed the experimental diets for another 14 days. Twenty-four hours urine was collected for analysis of urinary glucose. On the 14th day, blood was collected by cardiocentesis to obtain serum, and the liver was extirpated; both specimens were stored at $-30^{\circ} \mathrm{C}$ until analysis could be performed.

Table 1 shows the compositions of the diets. The diet without addition of the tea samples was given to the control animals (with STZ diabetes). The additions 
Table 1. Compositions of experimental diets.

\begin{tabular}{lrrrr}
\hline Ingredients & Control & Maccha & $\begin{array}{r}\text { Maccha-extract } \\
(\mathrm{g} / 100 \mathrm{~g} \text { diet })\end{array}$ & Mate-extract \\
\hline Casein & 20.0 & 20.0 & 20.0 & 20.0 \\
Corn oil & 5.0 & 5.0 & 5.0 & 5.0 \\
Sucrose & 50.0 & 50.0 & 50.0 & 50.0 \\
Corn starch & 15.3 & 12.3 & 14.3 & 14.3 \\
Cellulose powder & 5.0 & 5.0 & 5.0 & 5.0 \\
AIN-76 mineral mix* & 3.5 & 3.5 & 3.5 & 3.5 \\
AIN-76 vitamin mix* & 1.0 & 1.0 & 1.0 & 1.0 \\
Choline chloride & 0.2 & 0.2 & 0.2 & 0.2 \\
Maccha (Powdered green tea) & - & 3.0 & - & - \\
Maccha alcohol extract & - & - & 1.0 & - \\
Mate tea alcohol extract & - & - & - & 1.0 \\
\hline
\end{tabular}

*J. Nutr., 107, 1340 (1977). Supplied by Oriental Yeast Co., Ltd., Tokyo, Japan.

were made so as to bring the crude catechin content in the diets to be substantially equal among the groups.

All animals were fed the $25 \mathrm{~g}$ /day diet as a meal and given free access to tap water.

Analytical methods. The following indices were determined to evaluate severity of the diabetic conditions: urinary glucose excretion and fasting blood sugar, measured with a V-glucase kit (Nissui Pharmaceutical Co., Ltd., Tokyo, Japan); and triglyceride and total cholesterol concentrations, determined with triglyceride and cholesterol E test kits (Wako Pure Chemical Industries, Ltd., Osaka, Japan), respectively. Serum and liver concentrations of thiobarbituric acidreactive substances (TBARS) were measured according to the methods of Yagi [9] and Ohkawa et al. [10] as a parameter of disorders in the body caused by STZinduced oxidation. The serum concentration of uric acid, a major antioxidant component in the body, was determined with a Uricadase kit (Nissui Pharmaceutical Co., Ltd., Tokyo, Japan); and liver nicotinamide adenine dinucleotide (NAD), by Kingenberg's method [11].

Statistical evaluation was performed by Student's $t$-test at $p<0.05$.

\section{RESULTS}

A control group comprising animals with STZ diabetes was provided for each of the test groups. Figure 1 shows changes in glucose excreted in the urine after administration of STZ. Urinary glucose, which serves as an indicator for sideration of experimentally induced diabetes, was markedly suppressed in the Maccha group when compared with its level in the control animals. We also observed that the alcohol extract of either Maccha or Mate tea decreased the excretion relative to the value for the control animals.

Figure 2 shows fasting blood sugar values (at $5 \mathrm{~h}$ after starvation) upon completion of the experimental period. These values were also decreased signifi- 

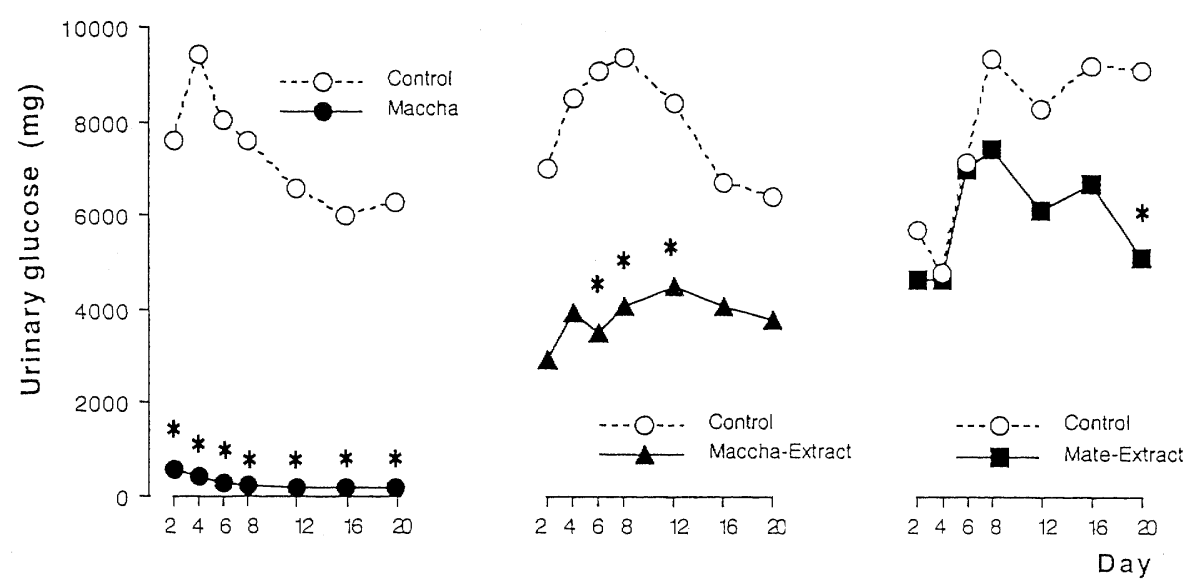

Fig. 1. Urinary excretion of glucose in rats fed the experimental diets. Values are mean of 8 rats. ${ }^{*}$ Significantly different from the control group $(p<0.05)$.

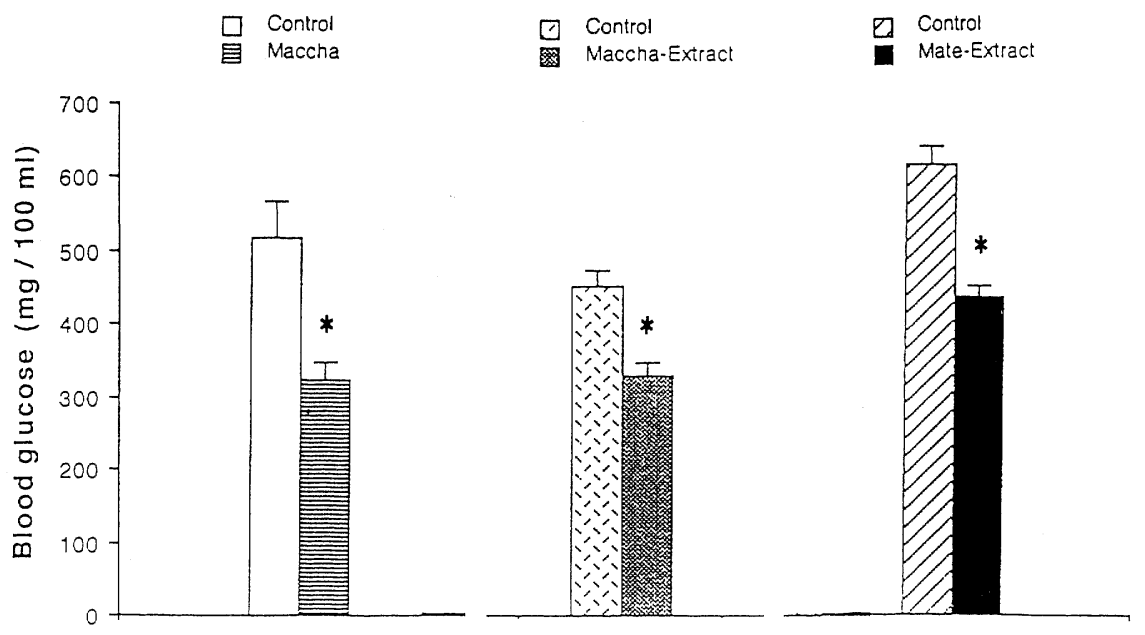

Fig. 2. Concentration of blood glucose in rats fed the experimental diets. Values are mean \pm SE $(n=8)$. ${ }^{*}$ Significantly different from the control group $(p<0.05)$.

cantly when compared with those of the control animals. Based on these findings, we concluded that the continuous intake of Maccha or the alcohol extract of Maccha or Mate tea markedly suppressed the onset of experimental diabetes. While there was a restraint in body weight gain in the control animals, no such restraint was observed in either the Maccha group or the two extract groups (data not shown).

Table 2 shows the serum triglyceride and total cholesterol concentrations. Both were significantly lower in those animals given Maccha or either of the two extracts than in the control animals with diabetes. The intake of these substances 
Table 2. Concentrations of triglyceride and total cholesterol in serum of rats fed the experimental diets.

\begin{tabular}{lcc}
\hline Groups & $\begin{array}{c}\text { Triglyceride } \\
(\mathrm{mg} / 100 \mathrm{ml})\end{array}$ & $\begin{array}{c}\text { Total cholesterol } \\
(\mathrm{mg} / 100 \mathrm{ml})\end{array}$ \\
\hline Control & $275 \pm 6.5^{*}$ & $131 \pm 5.2$ \\
Maccha & $90 \pm 8.3^{\mathrm{a}}$ & $102 \pm 3.1^{\mathrm{a}}$ \\
Control & $253 \pm 3.2$ & $104 \pm 3.9$ \\
Maccha-extract & $76 \pm 6.8^{*}$ & $74 \pm 3.6^{\mathrm{a}}$ \\
Control & $247 \pm 7.9$ & $122 \pm 2.1$ \\
Mate-extract & $125 \pm 7.1^{\mathrm{a}}$ & $99 \pm 2.5^{\mathrm{a}}$ \\
\hline
\end{tabular}

* Mean \pm SE of eight rats. ${ }^{\text {a }}$ Significantly different from the control group at $p<0.05$.
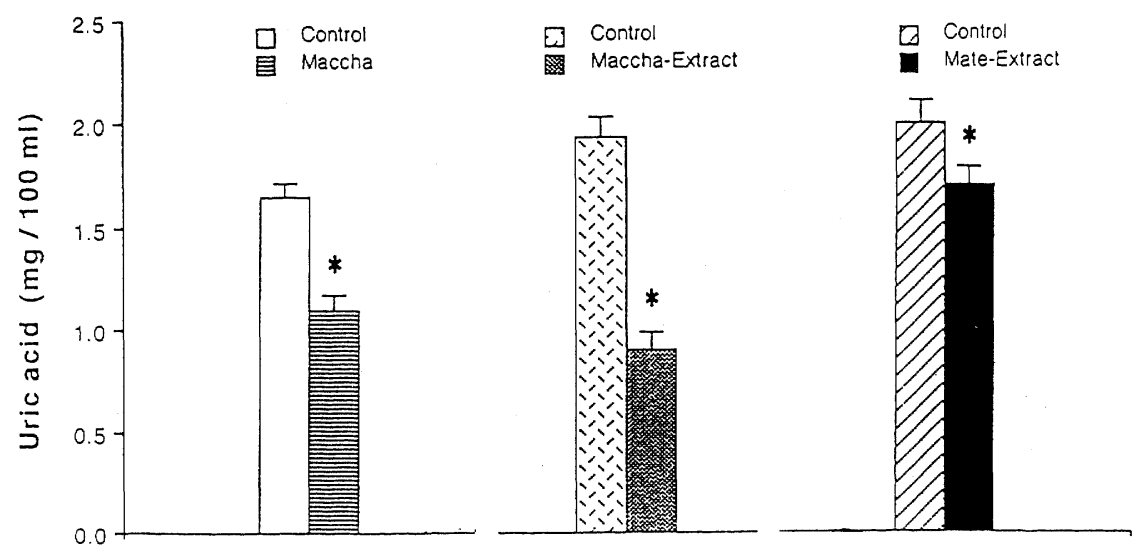

Fig. 3. Concentration of uric acid in serum of rats fed the experimental diets. Values are mean \pm SE $(n=8) .{ }^{*}$ Significantly different from the control group $(p<0.05)$.

Table 3. Concentration of TBARS in serum and liver of rats fed the experimental diets.

\begin{tabular}{lcc}
\hline Groups & $\begin{array}{c}\text { Serum } \\
(\mathrm{nmol} / \mathrm{ml})\end{array}$ & $\begin{array}{c}\text { Liver } \\
(\mathrm{nmol} / \mathrm{g})\end{array}$ \\
\hline Control & $7.8 \pm 0.3^{*}$ & $507 \pm 31.5$ \\
Maccha & $6.7 \pm 0.4^{\mathrm{a}}$ & $412 \pm 27.1^{\mathrm{a}}$ \\
Control & $7.9 \pm 0.2$ & $586 \pm 21.7$ \\
Maccha-extract & $6.9 \pm 0.2^{\mathrm{a}}$ & $460 \pm 25.2^{\mathrm{a}}$ \\
Control & $7.0 \pm 0.2$ & $475 \pm 35.9$ \\
Mate-extract & $6.5 \pm 0.2^{\mathrm{a}}$ & $282 \pm 22.3^{\mathrm{a}}$ \\
\hline
\end{tabular}

${ }^{*}$ Mean \pm SE of eight rats. ${ }^{a}$ Significantly different from the control group at $p<0.05$.

assumedly acted effectively against the elevation of serum lipids caused by experimental diabetes.

Figure 3 shows serum uric acid concentrations. The values were decreased significantly by the intake of Maccha or either of the two extracts.

Table 3 shows serum and liver TBARS concentrations. The serum TBARS Vol. 20, No. 1, 1996 


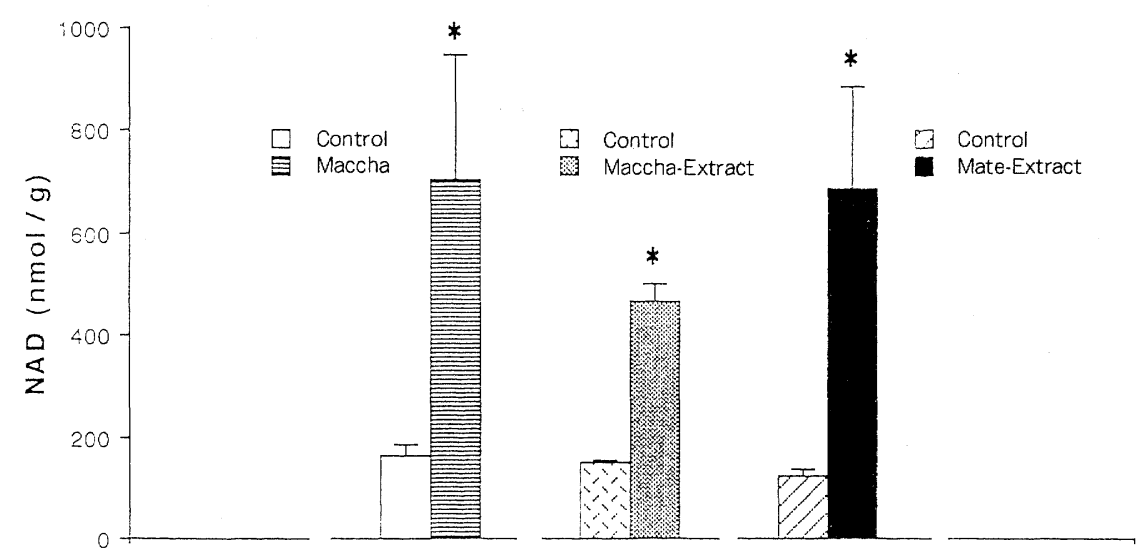

Fig. 4. Contents of NAD in liver of rats fed the experimental diets. Values are mean $\pm S E$ $(n=8)$. *Significantly different from the control group $(p<0.05)$.

concentration was significantly lower in the Maccha group or either of the two extract groups when compared with its level in the control animals with diabetes. The liver TBARS concentration was also significantly decreased in the treated groups, particularly in the Maccha group and the Mate extract group.

Figure 4 shows liver NAD. Liver NAD markedly decreased upon the onset of STZ diabetes, but the decrease was suppressed by the intake of Maccha or either of the two extracts.

\section{DISCUSSION}

Experimental animals with alloxan (AX)- or STZ-induced diabetes serve as models for human IDDM. In recent years, IDDM is considered to be caused by the selective destruction of pancreatic $\beta$ cells by an autoimmune mechanism [2]. In other words, the onset of IDDM is triggered by free radicals released directly from immunocytes such as macrophages or by those formed in Langerhans' islands by the action of cytokines released from immunocytes $[1,12]$. In view of the above, attempts have been made lately to prevent or suppress the development of IDDM by administering a free-radical scavenger to model animals with AX or STZ diabetes or hereditary I-type diabetes. Reports of delayed development of diabetes in animals with hereditary spontaneous diabetes by administration of chemicals that act as free-radical scavengers have been made; e.g., on the effect of probucol in BB rats by Drash et al. [13] and in NOD mice by Fukuda et al. [14]. Yamada et al. [15] administered nicotinamide to NOD mice and observed suppressed sideration based on blood sugar and histological study of the Langerhans' islands. Mendola et al. [16] reported that the onset of diabetes and elevation of blood sugar were suppressed and that histologically observed destruction of $\beta$ cells could be prevented by administering an iron chelating agent to animals with STZ 
diabetes or hereditary diabetes. Most of these reports dealt with experiments using drugs, and hardly any attention was paid to food components having antioxidant activity. Foods or their components have not been fully studied to date to elucidate their activity to suppress or prevent sideration of diabetes. In the present study, we studied the effects of Maccha and alcohol extracts of Maccha and Mate tea, from which catechins can be ingested relatively directly, for possible suppression of diabetic symptoms.

We found that continuous oral administration of these substances prior to dosing with STZ effectively suppressed urinary excretion of glucose and lowered fasting blood sugar and that these substances suppressed sideration of diabetes experimentally induced by STZ. The tea or tea extracts also restrained the manifestations accompanying diabetes, such as the elevations of serum lipids and serum and liver TBARS, and alleviated the disorders caused by oxidation in the body. These findings suggest that continuous intake of some food components or foods containing such components achieves effects comparable to those obtained by administration of the drugs discussed above. Uric acid, a major antioxidant compound in blood, increased in the control animals, contrary to general expectations, and decreased in the treated groups. The uric acid level has been observed to rise temporarily by administration of STZ [17], which elevation is considered to occur as compensation for the disorders in the body caused by oxidation. The components of tea leaves assumedly mitigated the oxidation-induced disorders and lowered the uric acid level. Although uric acid is strongly antioxidative in the body, it also causes hyperuricemia and gout. Elevation in blood uric acid is therefore considered to be undesirable. Thus, intake of tea components is all the more effective in this respect.

The intake of Maccha, as compared with that of the alcohol extracts of Maccha and Mate tea, was particularly effective in suppressing urinary glucose excretion. These substances were observed to also decrease serum and liver TBARS concentrations significantly. The effect of Maccha and the two alcohol extracts in suppressing sideration of diabetes was considered attributable to the compounds contained in these, particularly to the catechin antioxidants. Typical catechins contained in Maccha and Mate tea include epigallocatechin, epicatechin, epigallocatechin gallate, and epicatechin gallate [18]. Okuda et al. [19] studied the in vitro effect of polyphenol compounds on lipid peroxidation in rat liver mitochondria and microsomes, and noted an extremely potent suppressive effect of epigallocatechin gallate compared with that of $\alpha$-tocopherol. Namiki and Osawa [20] conducted an in vitro study using rat liver microsomes and rabbit erythrocyte ghosts, and found that catechin gallates were effective in suppressing lipid peroxidation similarly as $\alpha$-tocopherol and BHA and in scavenging superoxide radicals. However, these findings were obtained by in vitro experiments and not in vivo ones. In contrast, our results show that the components of tea leaves fed to the animals were absorbed and manifested a direct effect on the living body. In short, our study demonstrated their in vivo activity. Radicals are involved in exacerba-

Vol. 20, No. 1, 1996 
tion or onset of complications of either IDDM or NIDDM. Our data suggest that these foods and their components may possibly be utilized to prevent diabetes and improve its clinical conditions.

However, we did not observe any obvious correlation between the extent of suppression of sideration of diabetes produced by the test substances and the serum or liver TBARS concentration. Further studies to investigate the relation between the dose levels of the test substances and the extent of suppression of sideration and to identify the active component are warranted.

Liver NAD markedly decreased upon onset of STZ diabetes, but the decrease was suppressed by intake of Maccha or either of the alcohol extracts of Maccha and Mate tea. NAD is a coenzyme of oxidoreductases and plays an important role in restoring cell function after STZ administration by serving as a substrate for poly(ADP-ribose) synthesis preceding DNA repair after DNA cleavage caused by STZ-induced radicals $[21,22]$. This suggests that the intake of Maccha or the alcohol extract of Maccha or Mate tea is effective not only in maintaining metabolic reduction but also in restoring functions of the disordered cells.

Urinary glucose excretion was markedly suppressed by the intake of Maccha alone. In addition to the antioxidant property of the components of tea leaves, fibers contained in Maccha are assumed to have enhanced the effect by inhibiting intestinal absorption of glucose.

The expenses of the present study were partially financed by the "1994 Research Grant" of the Skylark Food Science Research Institute.

\section{REFERENCES}

1. Brownlee, M. (1993): Glycation products and the pathogenesis of diabetic complications. Diabetes Care, 15, 1835-1843.

2. Satoh, J., Sagara, M., Takahashi, K., and Toyota, T. (1993): The role of free radical in $\beta$ cell destruction in insulitis lesion. J. Act. Oxyg. Free Red., 4, 142-148.

3. Anderson, W.J., and Ward, K. (1978): Long-term effects of high-carbohydrate, high-fiber diets on glucose and lipid metabolism; a preliminary report on patients with diabetes. Diabetes Care, 1, 77-82.

4. Jenkins, D.J.A., Leeds, A.R., Gassull, M.A., Cochet, B., and Alberi, K.G.M. (1977): Decrease in postpr andial insulin and glucose concentrations by guar and pectin. Ann. Intern. Med., 86, 20-23.

5. Jenkins, D.J.A., Wolever, T.M.S., Leeds, A.R., Gassull, M.A., Haisman, P., Dilawari, J., Goff, D.V., Metz, G.L., and Alberi, K.G.M. (1978): Dietary fibers, fiber analogues and glucose tolerance; importance of viscosity. Br. Med. J., 1, 1392-1394.

6. Bolton, R.P., Heaton, K.W., and Burroughs, L.F. (1981): The role of dietary fiber in satiety glucose, and insulin; studies with fruit and fruit juice. Am. J. Clin. Nutr., 31, 738-741.

7. Ceriello, A., Giugliano, D., Quatraro, A., Donzella, C., Dipalo, G., and Lefebvre, P.J. (1991): Vitamin E reduction of protein glycosylation in diabetes. Diabetes Care, 14, 68-72.

8. Pritchard, K.A., Jr., Patel, S.T., Karpen, C.W., Newman, H.A.I., and Panganamala, R.V. (1986): Triglyceride-lowering effect of dietary vitamin $\mathrm{E}$ in streptozotocin-induced diabetic rats. Diabetes, 35, 278-281.

9. Yagi, K. (1984): Assay of modes of biological damage imposed by $\mathrm{O}_{2}$ and reduced species. Assay for plasma or serum. Methods Enzymol., 105, 328-331. 
10. Ohkawa, H., Ohishi, N., and Yagi, K. (1979): Assay for lipid peroxides in animal tissues by thiobarbituric acid reaction. Anal. Biochem., 95, 351-358.

11. Kingenberg, M. (1974): Nicotinamide adenine dinucleotides (NAD, NADP, NADH, NADPH) spectrophotometric and fluorimetric methods, in Methods of Enzymatic Analysis, Vol. IV, ed. by Bergmeyer, H.U., Academic Press, New York, pp. 2045-2059.

12. Rabinovitch, A. (1994): Immunoregulatory and cytokine imbalance in the pathogenesis of IDDM, therapeutic interventions by immunostimulation? Diabetes, 43, 613-621.

13. Drash, A.L., Rubert, W.A., and Borquaye, S. (1988): Effect of probucol on development of diabetes of BB rats. Am. J. Cardiol., 62, 27B-30B.

14. Fukuda, M., Ikegami, H., Kawaguchi, Y., Fujioka, Y., and Ogihara, T. (1992): Antioxidant inhibits generation of free radicals in islet cells and prevents diabetes in NOD mice. Diabetes, 41, 35A.

15. Yamada, K., Nonaka, K., Hanafusa, T., Miyazaki, A., Toyoshima, H., and Tarui, S. (1982): Preventive and therapeutic effects of large-dose nicotinamide injections on diabetes associated with insulitis. Diabetes, 31, 749-753.

16. Mendola, J., Wright, J.R., Jr., and Lacy, P.E. (1989): Oxygen free-radical scavengers and immune destruction of murine islets in allograft rejection and multiple low-dose streptozotocin induced insulitis. Diabetes, 38, 379-385.

17. Wada, M., Tadokoro, T., Nakajima, H., and Maekawa, A. (1992): Influence of streptozotocin administration on blood uric acid levels in rats. Med. Biol., 124, 257-260.

18. Balentine, D.A. (1992): Manufacturing and chemistry of tea, in Phenolic Compounds in Food and Their Effects on Health I, ed. by Ho, C., Lee, C.Y., and Huang, M., American Chemical Society, Washington, D.C., pp. 102-117.

19. Okuda, T., Kimura, Y., Yoshida, T., Hatano, T., Okuda, H., and Arichi, S. (1983): Studies on the activities of tannins and related compounds from medicinal plants and drugs. I. Inhibitory effect on lipid peroxidation in mitochondria and microsomes of liver. Chem. Pharm. Bull., 31, 1625-1631.

20. Namiki, M., and Osawa, T. (1986): Antioxidants/Antimutagens in foods. Basic Life Sci., 39, $131-142$.

21. Uchigata, Y., Yamamoto, H., and Okamoto, H. (1982): Protection by superoxide dismutase, catalase and poly(ADP-ribose) synthetase inhibitors against alloxan and streptozotocininduced islet DNA strand breaks and against the inhibition of proinsulin synthesis. J. Biol. Chem., 257, 6084-6088.

22. Behme, M.T. (1995): Nicotinamide and diabetes prevention. Nutr. Rev., 53, 137-139. 